\title{
SDN Low Latency for Medical Big Data Using Wavelets
}

\author{
Fadia Shah, Jianping Li, Raheel Ahmed Memon \\ School of Computer Science and Engineering, University of Electronic Science and \\ Technology of China, Chengdu 611731, China \\ fadiashah13@yahoo.com, ipli2222@uestc.edu.cn, raheelmemon@iba-suk.edu.pk
}

\begin{abstract}
New era is the age of $5 \mathrm{G}$. The network has moved from the simple internet connection towards advanced LTE connections and transmission. The information and communication technology has reshaped telecommunication. For this, among many types of big data, Medical Big Data is one of the most sensitive forms of data. Wavelet is a technical tool to reduce the size of this data to make it available for the user for more time. It is also responsible for low latency and high speed data transmission over the network. The key concern is the Medical Big Data should be accurate and reliable enough so that the recommended treatment should be the concerned one. This paper proposed the scheme to support the concept of data availability without losing crucial information, via Wavelet the Medical Data compression and through SDN supportive architecture by making data availability over the wireless network. Such scheme is in favor of the efficient use of technology for the benefit of human beings in the support of medical treatments.
\end{abstract}

Keywords: Medical Big Data, Cache, SDN, Wavelets, Compression, Low Latency.

\section{Introduction}

With every passing day, there is more increase in mobile phones and their applications, smart devices, sensors, automated systems; therefore, the world has become a global village not only for humans but also for machines. The wireless network is modified from connection of devices and web services to the internet of things (IoT). Many IoT [26] devices are working under Medical Big Data support [14, 17]; many healthrelated applications are developed and are automatically working without human involvement [23]. The smart phone, with a Heterogeneous environment (HetNet) has a number of smart automatic applications. Since there is a remarkable increase in Machine-toMachine (M2M) communication as well as
Human-to-Machine (H2M) communication, this thing makes the Medical Big Data a really very big data. For a proper network traffic the flow of Medical Big Data; and Network traffic engineering [1, 23] for this MBD transmission to the proper destination is very vast research topic in many directions.

In this regard there are the data reliability, maintenance, updating and modification as the key concerns, since Medical history is the most important requirement at any time interval for a proper diagnosis. Medical Big Data is available in many forms like X-Rays, CT scan, MRI, ECG, EEG and many more. This all data is considered for every individual due to the fact that medical related decisions are based upon 
this. Missing a single piece of information can mislead the proper treatment. When this data is made available over the internet models, for example the cloud technologies, its reliability is the most important issue; even security is another concern. Just like in developing countries E-Health is a very popular technology. More work is still going on to make these things work smoothly under one platform.

Now a day's more devices are made available online. The automated systems collect information and update it that makes it a huge network traffic and the most useful information to be available for more time. The architecture which supports huge and fast network traffic is Software Define Network (SDN) [5] for 5G technology. It is flexible, job-oriented, supportive and capable to tolerate the high bandwidth utilization. It has two separate planes $[1,2]$. Such scheme is suitable for huge number of devices with a variety of operating systems, but all work efficiently under one platform. The high speed network with less time span is very much favourable for the upcoming latest technologies.

In future there will be much more load of medical data over the network for the companies concerned with technical health. In advanced countries, there are organizations working on health data management systems in more refined and sophisticated ways.

The Fig. 1 shows SDN architecture indicating separate levels of SDN for network management by dividing the system into two basic modes. They are connected by an interface. The requests are fulfilled by Controlled Plane. And the data plane focuses only on the information. More and more devices are involved to the network and their efficiency is tried to entertain at the maximum level.

The remaining part of this paper is organized as follows: Section 2 presents the brief introduction of wireless network architecture with SDN and information processing. It also relates the work with SJCMS | P-ISSN: 2520-0755 | Vol. 1 | No. 1 | (C) 2017 Sukkur IBA advancements made so far in order to increase system efficiency. Section 3 clearly states the problem definition and its importance. Section 4 states the proposed architecture along-with its layout, compatibility and components that emphasize its importance for adaption. Section 5 shows the results and Section 6 is for conclusion and limitations.

\section{Background}

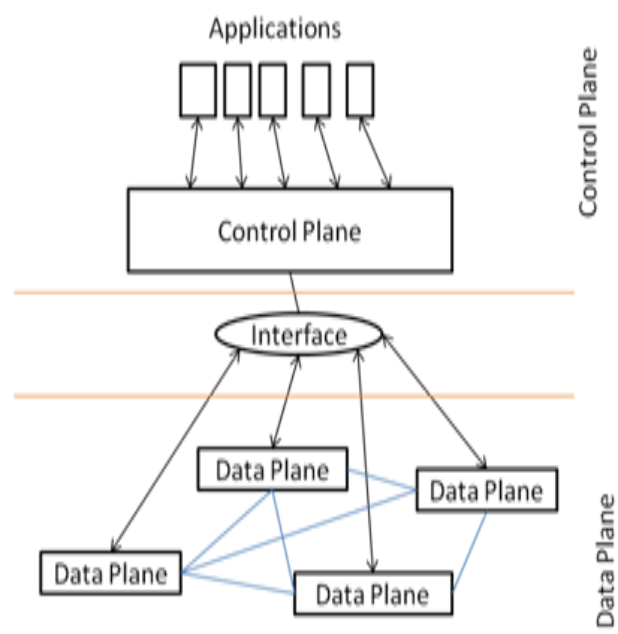

Figure. 1: SDN Architecture Indicating Two Planes

Soon after the discovery of internet and device sharing, cloud technologies became a very interesting and vital topic and area of research. It is still a very informative and innovative concern for new researchers. When it is information over the cloud, the key concern is the data availability and reliability. Precisely, the Medical Big Data availability is the most important concern. Its storage and consultation cannot be maintained manually.

A. Motivation: Big Data is concerned with huge increase in the data and since any information related to medical sciences compels the proper and necessary treatment directions, so its every bit can't be negligible. The cloud mobile network is the hot favourite area of discussion in practical technology lives $[7,11,20]$. For smallest activities, human 
beings are being replaced by machines. This thing has many advantages and disadvantages at times; but in any case the concern is to focus on the information saving and utilization schemes. For this big data over wireless network, this concept further improved the management of a huge number of users and originated SDN more popular which redirect control and data plane separately. As a result now we are having tones of data containing the least relevant and proper information. MBD Information is a key concern in diagnosis and the suitable treatment of a patient [20]. The basic purpose is to transfer medical information from the source to destination within a remarkable time, such that there is no missing information and it is useful at both ends. The information communication architectures are now being developed based upon Information Centric Network (ICN) [13, 19], but, unfortunately, the SDN architecture cannot directly support ICN operations. Network Virtualization (NV) [2] and Network Function Virtualization (NFV) extended the utility of network beyond traditional WMN [15, 16]. The ICN are content-based networks that rely data flow from IP to named-based content routing [10]. The SDN Controller [6] can provide the network with programmable functions, such as a load balancer, Network Address Translation (NAT), firewall and specialpurpose routing protocol [10]. It is difficult to access MBD all so efficiently and easily with ordinary schemes. So for new network architectures and schemes, network efficiency and reliability is being improved gradually.

B. Related Works: For heavy network traffic, especially wireless network, cache management works efficiently for MBD over Heterogeneous Networks. This is also useful for medical Big Data. Cache is the temporary memory which can store MBD in the form of image and video files from one source to anywhere in the network. The Information Centric Networks structure identifies and is aware of the context of a request. The LTE Radio link [7] connection is strong platform and acts as a backbone but sometimes its reliability is doubtful; thus the cache aware networks [5] ensure content availability.

Network is more effective using SDN via NFV [11] that shares required network functions virtually and globally, extending network utility via MBD content management [10,18]. An Information Centric Network node has two basic functions: a) caching function which caches a data. B) a name-based routing function that routes a user request and its corresponding response based on the name of the data object.

Fig 2. Describes the basic network infrastructure where Evolved Packet Core (EPC) is the core network connection and Long Term Evolution (LTE). Basic Network Infrastructure backbone and radio link LTE is responsible for increasing speed and capacity together with core network. Such infrastructure is very much helpful in MBD transfer via SDN [11] for upcoming technology. MBD from Controller to Base Station (BS) and nodes eNodeB, a variety of devices are receiving the requested MBD.

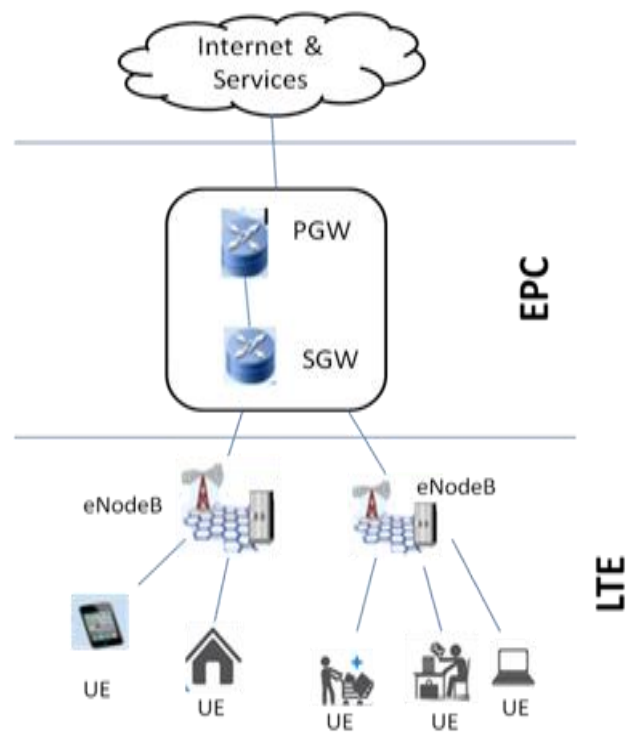

Figure. 2: MBD Storage in Wireless Network 
Most of the devices are working on different platforms, hardware and software. The main concern is to entertain every request and maximize the cache hit ratio via Openflow protocols [10] to ensure valuable communications. To share traffic load in tangible time, Coordinating cache [9] share their MBD status with some neighbouring cache. Specific content delivery networks [9].

$$
\left(\text { Effeciency) } H_{R}=1-M_{R}\right. \text { (1) }
$$

Where $\mathrm{H} \mathrm{R}$ is the Hit rate and MR is the Miss rate. The equation 1 indicates the efficiency of the medical data related wireless network can be improved if the miss rate is reduced. For load balancing it is most important thing to access MBD smoothly without blocking the network flow. In the past it was the serious problem of redundancy [7, 8 ] but now instead of replicating data, only the cache status is coordinated, dynamic cache allocation [7], MBD via wavelet.

\section{Problem Definition}

In this smart world, we are millionaires in data but starving in information. MBD in the form of X-Ray image, MRI, CT scan, ECG and video is stored over wireless network. Information centric network targets information instead of IP address. For this purpose, the system medical data in the form of information must be shared to get the right access. It needs proper storage for further efficient access strategies. The actual problem is to digitize the data and make it available to only the concerned consultant. The patient doesn't need to keep the loads of medical history in the form of manual files; but now to use the advance technology to facilitate healthcare improvement schemes. The actual scheme is cooperative cache that share their status to facilitate network. There are a number of cache schemes implemented to improve the system latency. For example, the increase in size of network cached, implement on-path and off-path cache schemes, distributed cache architecture, coordinating and noncoordinating cache, content popularity and network traffic. Along with these all facilitating network schemes, the concern is which data to store. The network infrastructure is vast and versatile. The main problem is how to store this critical medical big data on wireless network to access it in least latency. To activate more devices and applications over the heavily functional network, conversion of the Information Centric Network to Information Critical Network is the key concern in the proposed system.

\section{Architecture}

In the proposed system, the coordinating and dedicated cache will support the query fulfilment up to as much maximum level as possible. As the network is enriched with a huge number of cache all over the network, the Medical Big Data is set available at a few nodes but not on all the nodes over the network. The relevant data is made available on some of the cache intelligently. Since, the network Controller has all information of the network, the dedicated cache reduces the Controller load too. The Core network with centralized cache and for Ad-Hoc network coordinated cache. The Medical Big Data can be observed under two schemes. One type of data which utilize more bandwidth over the network like multimedia or video data, such type is the CT scan, MRI, etc. Another type of medical-related data is image file and utilizes low bandwidth like the X-Ray, ECG file, Consultant report, etc.

A. Design Layout and Compatibility: The distinct feature of the wireless network architecture considered in this paper is to reduce latency time by utilizing cache by an intelligent manner. It means to apply the medical data availability on the nearest or early redirected cache. For a popular BS, the number of cache hit rate is more due to the 
number of user equipment's as compared to the cache at isolated edge. Make some cache as the dedicated cache for medical data availability; and some cache as the content aware cache.

The size reduction can be done using Wavelets which is an excellent tool to efficiently reduce the size of Medical Big Data in a very reliable manner. It truncates more useless part of the data and makes available the information containing bites. Through this way there is no more huge size of the information and this way the required information can be stored over the network and can be accessed back without utilizing more bandwidth.

The cache architecture is no doubt coordinating and shares data but not every cache is a status-sharing cache. The coordinating cache architecture is quite familiar and very well-known as before. It is emphasized that the neighbouring cache of every high bandwidth utilization data cache is the coordination cache. This reduces the network overload and flexible traffic management. Thus most of the requests are fulfilled thus improving cache hit rate.

B. Methodology: The Content Centric Network is not aware of IP addressing to fulfil requests efficiently and effectively. More the miss rate means the poor network efficiency. With normal network traffic with a limited bandwidth, more miss rate will increase load to receive request, time to search the required data and acknowledgements (most of the scenario).

The medical big data digitization will make the data from ordinary and sensitive file to a refined secure digital form. For example, the considered images of X-ray file format are selected. It can be seen that the normal X-ray image represents the current state of a patient. The chest X-ray is showing the lungs condition, the spots at different places and the dental X-ray shows the status of oral cavity and gums. Similarly the skull X-ray also contains the critical information of head region. It is not possible to make such information available all the time, so the advanced way is to make them available over the wireless network, store it in the cache and via SDN making cache coordinating and sharing the status of dedicated cache all over the network when needed.

Ordinary systems make such type of situations end at the system malfunction at any

Table 1: Original and Wavelet Compressed Image Formation

form like deadlock or snail network working.

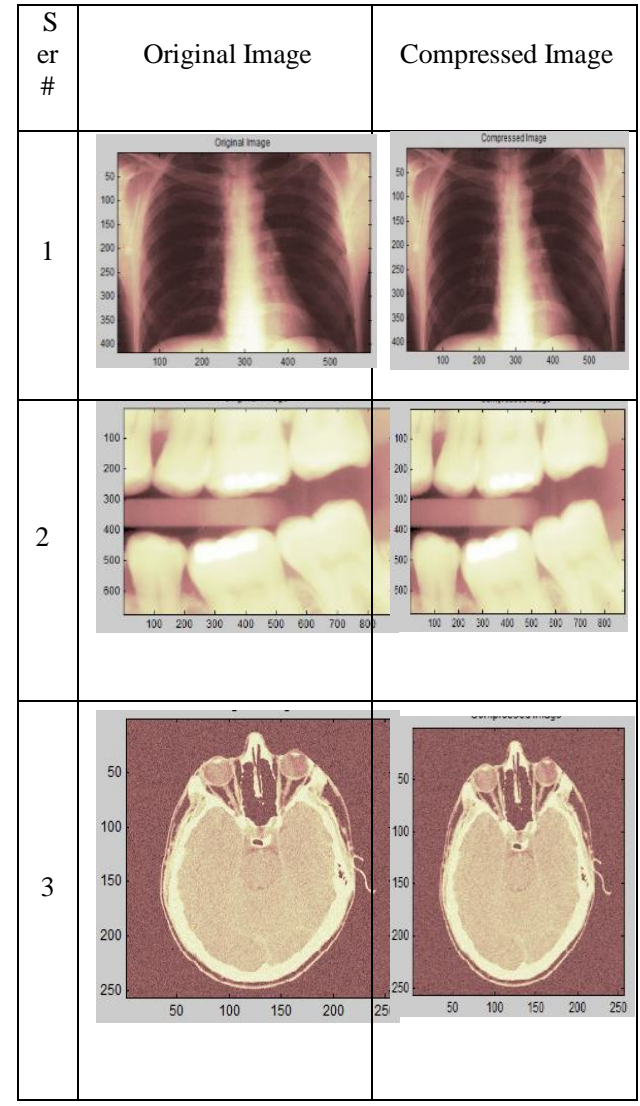

Table 1 shows the images before and after the image compression is done by using Wavelets Compression. It can be seen that the image 
quality is almost the same as in the original image. The three different types of images were selected and processed and were found almost as the same image. The difference in the images is so negligible that we can rely on the compressed image for decision making. So such type of scheme for compression is applicable on SDN technology. The network for Medical Big Data accessing and functioning efficiency can be observed by the time utilization on average, this can be calculated by:

$$
T_{\text {avg }}=h c+(1-h) M
$$

Equation 2, where Tavg is average time, $\mathbf{h}$ stands for hit rate, $\mathbf{c}$ stands for cache, (1-h) is for miss rate, $\mathbf{M}$ stands for miss penalty. The requesting device gets the hit ratio for requested image. More the number of devices needs more efficiency to maintain the quality of network utilization. MBD available at any of the network edges, for latest technologies it is being struggled to reduce the latency up to $1 \mathrm{~ms}$.

By this type of combination of Medical Big data by Wavelets Compression and this data flow over the network by SDN architecture improves the network cache hit ratio but when the request is not fulfilled, it is redirected to the other destination instead of declaring the failed query response. This ensures least request dropout. It means less miss ratio. The MBD Coordinated Cache in terms of Euclidian Distance is a good approach for accessing MBD in a better way to ensure data availability. It is therefore,

(3)

$$
\operatorname{dist}(x, y),(a, b)=\sqrt{(x-a)^{2}+(y-b)^{2}}
$$

From user equipment to content directed cache, the Euclidian distance including noise can be determined by equation 3. For the proposed system, dedicated cache (where medical wavelet compressed data is residing) is determined at the most busy base stations, determining the more number of hits, the coordinating cache (which share the status and path of medical data cache) will be the average hit rate cache. This coordinating cache will be determined by Euclidian Distance. It will share the status from a remarkable distance from other cache.

\section{Results}

The wireless network without coordinating cache architecture for Medical Big Data with heavy network shows poor performance and less hit rate. Even if the medical data availability is made possible at different dedicated terminals; involving the Controller to dedicated cache and/or cache, and also the dedicated cache to cache coordination. Requests are thus redirected to only the concerned cache. This will increase system efficiency and improve the system latency time. The systems developing under SDN architecture with cache support are either coordinating cache based or dedicated cache based. But for Medical Big Data using this dedicated, coordinating cache, the latency reduction with proper information access improves the network efficiency. Such system supports the medical smart devices; wavelets ensure the minimization of data loss and data reliability in time and frequency domains. So this scheme is efficient in many aspects when implemented.

The graph determines the change in network performance when we change the number of cache for Medical Big Data availability. This is with general data orientation. It shows here blue line means hit rate for request response hit rate and green line means cache status sharing with neighbouring and it improved the performance. For about 200 cache the system performance is tested.

The system hit rate is increased when the heavy video medical data compressed via Wavelet compression is kept at farther cache and dense and more image Wavelet compressed data is stored near to user equipment. The system is tested in three ways. 
Test 1: When there is simple request response from cache assuming performance is 30 percent improved.

Test 2: When the cache redirects request towards SDN controller assuming performance is 55 percent improved.

Test 3: When there is dedicated coordinating cache assuming performance is 75 percent improved.

The increasing in number of cache reduces network load, the coordinating cache further reduces the network load by sharing the status and dedicated distributed coordinating cache not only shares its status but the dedicated cache has the status of subnet or neighbouring cache. This means dedicated Medical Big Data storage in cache. From equation 2 and 3, applying K- Nearest Neighbour algorithm, the system efficiency is noticeably improved and is represented by the following graph. Here, the blue lines show the best performance due to coordinated cache and easily approachable Medical Big Data for user equipment.

Systems focusing MBD via advance LTE telecommunication systems are most concerned systems. It is tried to develop the systems which are most facilitating for the patient-consultant relationship. It will reduce decision time, towards least complications and

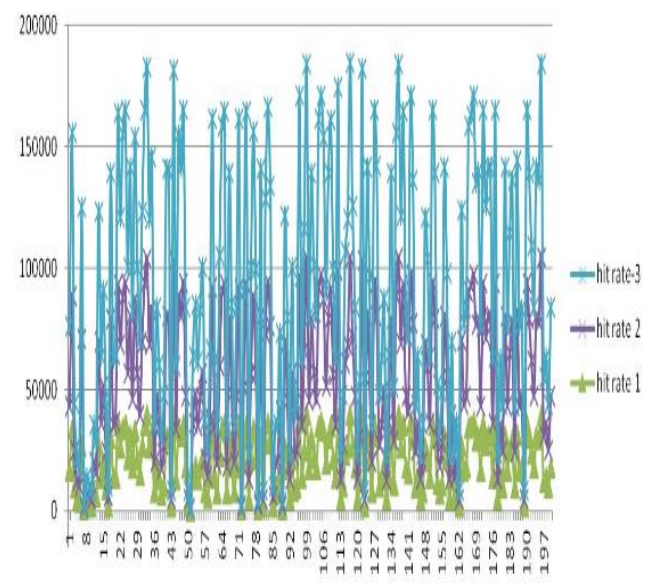

Figure. 3; Medical Big Data for Huge Network Coordinating Cache improve early diagnosis. In network dedicated coordinated content cache in distributed and wireless network systems is a smart scheme which can improve information retrieval while ensuring quality management. This is the backbone of health-based ideal systems.

\section{Conclusion}

Wireless Network technologies are improving the quality of life in different ways. The health quality improvement is a basic need for a productive society. For this concern, the medical big data availability from patient report generation to consultant is an important task. Using SDN technology and high speed network bandwidth utilization, the tele-health systems are being developed which the aim to maintain all medical health related all data for a proper diagnosis.

Keeping wireless technology as a backbone, SDN as a service-oriented technology, patient as a data-generating source, the low latency approach by reducing the size of medical data, its availability on distributed, dedicated and coordinating cache will help the treatment technologies in coming days.

\section{Limitations and Future Work}

The proposed system provides an efficient approach for MBD in network cache systems via distributed coordinating cache scheme. It is efficient to access information via considering low latency and system reliability. However, what data to exactly store, how to maintain data quality and storing more data within a limited cache, is still to be explored.

\section{Acknowledgements}

This paper was supported by the National Natural Science Foundation of China (Grant No. 61370073), the National High Technology Research and Development Program of China (Grant No. 2007AA01Z423), the project of Science and Technology Department of Sichuan Province. 


\section{References}

[1] Ian F. Akyildiz, Ahyoung Lee, Pu Wang, Min Luo, Wu Chou, "A roadmap for traffic engineering in SDN-OpenFlow networks." Computer Networks, ELSEVIER, 2014.

[2] Carlos J. Bernardos, Antonio de la Oliva, Hao Jin, Luis M. Contreras, Juan Carlos Zúñiga “ An Architecture for Software Defined Wireless Networking", IEEE, 2014.

[3] Zhenfa Wang, Lei Wang*, Xiangyu Gao, Ying Xia, Song Wang, "An Architecture of Content-Centric Networking over ProtocolOblivious Forwarding”, IEEE, 2015.

[4] Manisha Koranga , Nisha Koranga , “ Analysis on PageReplacement Algorithms with Variable Number of Frames”, IJARCSSE, Vol. 4, Issue 7, 2014.

[5] Xiaofei Wang, Min Chen, Tarik Taleb, Adlen Ksentini, Victor C.M.Leung, "Cache in the Air: Exploiting Content Caching and Delivery Techniques for 5G Systems", IEEE Communications Magazine, 2014.

[6] Francesco Pantisano, Mehdi Bennis, Walid Saad, and M' erouane Debbah, "Cacheaware User Association in Backhaul-Constrained Small Cell Networks", Fifth International Workshop on Indoor and Outdoor Small Cells, 2014.

[7] Ejder Ba c stug, Mehdi Bennis, Marios Kountouris and Mérouane Debbah, "Cacheenabled Small Cell Networks: Modeling and Tradeoffs" EURASIP, 2014.

[8] Mael Kimmerlin, Jose Costa-Requena, Jukka Manner, Damien Saucez, Yago Sanchez,"Caching Using Software-Defined Networking in LTE Networks", 2015.

[9] Shinae Woo, Eunyoung Jeong, Shinjo Park, Jongmin Lee, Sunghwan Ihm, KyoungSoo
Park," Comparison of Caching Strategies in Modern Cellular, MobiSys, "Ninth ACM conference on Emerging networking experiments, 2013 .

[10] Yanhua Li, Haiyong Xie, Yonggang Wen, Chi-Yin Chow and Zhi-Li Zhang, "How Much to Coordinate", IEEE, 2015.

[11] Won-Suk Kim, Sang-Hwa Chung, JaeWon Moon “ Improved content management for information centric networking in SDN-based wireless mesh network", Elsevier, 2015.

[12] Jose Costa-Requena $\{*\}$, Jesús Llorente Santos, Vicent Ferrer Guasch, Kimmo Ahokas, Gopika remsankar, Sakari Luukkainen, Ijaz Ahmad, Madhusanka Liyanage, Mika Ylianttila, Oscar López Pérez, Mikel Uriarte Itzazelaia, Edgardo Montes de Oca," SDN and NFV Integration in Generalized Mobile Network Architecture", IEEE, 2015.

[13] Suyong Eum, Masahiro Jibiki, Masayuki Murata, Hitoshi Asaeda, Nozomu Nishinaga," A design of an ICN architecture within the framework of SDN",ICUFN, IEEE, 2015.

[14] Carmen C. Y. Poon, Qing Liu, Hui Gao, Wan-Hua Lin, Yuan-Ting Zhang,"Wearable Intelligent Systems for E-Health“, JCSE, Vol. 5, No. 3, 2011.

[15] Khushpreet Kaur, Sheenam Malhotra ," Image Compression using HAAR Wavelet Transform and Discrete Cosine Transform", International Journal of Computer Applications, 2015.

[16] Xavier Giro-Nieto, and Jan Cornelis, "Wavelet Coding of Volumetric Medical Datasets", IEEE Transactions On Medical Imaging, 2003.

[17] Shivaputra, H.S.Sheshadri, V.Lokesha,"An Efficient Lossless Medical Image Compression Technique for Telemedicine 
Application", Computer Application: An International Journal (CAIJ) Vol-2, Ni1,February 2015.

[18] Sudeepti Dayal and Neelesh Gupta, "Region of Interest Based Compression of Medical Image using Discrete Wavelet Transform", International Journal on Computer Science \& Application (IJCSA) Vol 5, No 6, December 2015.

[19] Nuno Pombo, Nuno Garcia, Virginie Felizardo, Kouamana Bousson, "Big Data Reduction Using RBFNN: A Predictive Model for ECG Waveform for eHealth platform integration", IEEE HEALTHCOM 2014, Second International Workshop on Srvice Science for e-Health (SSH 2014).

[20] Vijay V, Gowri K, Velmurugan S, Dr.A.M. Basha, "Detection and Extraction of $\mathrm{P}$ Wave and $\mathrm{T}$ Wave in ECG to Improve Sensitivity for E-Health Monitoring”, IJCOTS, Vol-4, no-4, issue 02, March 2016.

[21] Ms. Shrutika S. Sawant, Dr. N. V. Dharwadkar, M.r. Sudodh. S. Ingaleshwar, “ A reiew on various Medical Image Compression Methods", IJIREEICE, Vol-4, Issue 1, January 2016

[22] Kokoulin Andrew, Dadenkov Sergey," Distributed Storage System For Imagery Data In Online Social Networks", July 2014

[23] Er. Shilpa Sachdeva, Er. Rajbhupinder Kaur,"A Review on Digital Image Compression Techniques", International Journal on Recent and Innovation Trends in Computing and Communication, ISSN: 2321-8169 Volume: 2 Issue: 7, IJRITCC | July 2014.

[24] WANG Hai, ZHENG Liyuan, “A Study on Wavelet Data Compression of a Real-Time Monitoring System for Large Hydraulic Machine",Vol.16 No.3 J. Comput. Sci. \& Technol. May 2001
[25] Muhammad Shahbaz, Aviral Kumar Tiwari \& Mohammad Iqbal Tahir, Analyzing timefrequency relationship between oil price and exchange rate in Pakistan through wavelets, Journal of Applied Statistics, 2015 Vol. 42, No. 4, 690-704, http://dx.doi.org/10.1080/02664763.2014.980784

[26] R.A. Menko, S. Visser, R. Janssen, M. Hettinga, T. Haaker, "APPLYING the STOF BUSINESS MODEL FRAMEWORK inEHEALTH-INNOVATION",ISBN:978-1-61208-

252-3,eTELEMED 2013 : The Fifth International Conference on eHealth, Telemedicine, and Social Medicine 